\title{
Non-exponentiality in electron transfer kinetics: Static versus dynamic disorder models
}

\author{
BIMAN BAGCHI \\ Solid State and Structural Chemistry Unit, Indian Institute of Science, \\ Bangalore 560012, India \\ Also at: Jawaharlal Nehru Centre for Advanced Scientific Research, Bangalore \\ e-mail: bbagchi@sscu.iisc.ernet.in
}

\begin{abstract}
Non-exponential electron transfer kinetics in complex systems are often analyzed in terms of a quenched, static disorder model. In this work we present an alternative analysis in terms of a simple dynamic disorder model where the solvent is characterized by highly non-exponential dynamics. We consider both low and high barrier reactions. For the former, the main result is a simple analytical expression for the survival probability of the reactant. In this case, electron transfer, in the long time, is controlled by the solvent polarization relaxation-in agreement with the analyses of Rips and Jortner and of Nadler and Marcus. The short time dynamics is also non-exponential, but for different reasons. The high barrier reactions, on the other hand, show an interesting dynamic dependence on the electronic coupling element, $V_{e l}$.
\end{abstract}

Keywords. Non-exponential dynamics; adiabatic surface; solvent reorganization energy.

\section{1._ Introduction}

Electron transfer reactions often show non-exponential kinetics. The probable cause for this non-exponentiality has been a subject of considerable discussion in the literature in the recent past. For biological electron transfer reactions, this nonexponentiality is usually atttributed to the existence of multiple configurations often observed in the biosystems, each with different activation energy and solvent environment. Understanding of this non-exponentiality, therefore, is highly non-trivial and requires the elucidation of reaction parameters for the different configurations. We shall not discuss this any further. Electron transfer reactions can be strongly nonexponential even in homogeneous phase, such as in liquids and glasses. Actually, the Sumi-Marcus theory ${ }^{1}$ was first formulated to explain such non-exponentiality observed in low or zero barrier photo-electron transfer reactions. Now, the nonexponentiality in Sumi-Marcus theory comes from the widening of the reaction zone due to the participation of a low frequency intra-molecular vibration in the electron transfer. The extent of this intrinsic non-exponentiality depends on the width of the reaction window determined by the ratio of the re-organization energies of the solvent and the vibrational modes and the degree of non-exponentiality is rather limited. There can, however, be another source of non-exponentiality in homogeneous systems. This is the non-Debye dielectric relaxation of the medium. In this article, we briefly discuss this issue. 
The attention on the role of non-Debye dielectric relaxation in the dynamics of electron transfer was triggered by an interesting article of McGuire and McLendon ${ }^{2}$ who found that electron transfer reaction in glassy glycerol was highly non-exponential. In particular, it was found that at short times, the electron transfer kinetics exhibits an interesting fractional power-law dependence on the characteristic dielectric relaxation time of the medium. At long times, the usual linear dependence was observed. These experimental results in turn motivated several interesting theoretical studies aimed at understanding the kinetics of electron transfer in non-Debye medium.

Rips and Jortner ${ }^{4}$ analysed the electron transfer reaction in the rigid glycerol by assuming a continuous distribution of relaxation times. They considered a medium characterized by the Davidson-Cole (DC) dielectric spectrum ${ }^{3}$ with the frequency $(\omega)$ dependent complex dielectric susceptibility function $(\varepsilon(\omega))$ given by

$$
\varepsilon(\omega)=\varepsilon_{\infty}+\left(\varepsilon_{0}-\varepsilon_{\infty}\right) /\left(1+i \omega \tau_{D C}\right)^{\beta}
$$

where $\varepsilon_{0}$ and $\varepsilon_{\infty}$ are the zero and infinite frequency dielectric constants, respectively, $\tau_{D C}$ is the characteristic (Davidson-Cole) relaxation time and the exponent $\beta$ is less than unity. For glycerol, $\beta$ has a nearly temperature independent value of about 0.6 . The Cole-Davidson expression provides a good fit for a large number of polar solvents, particularly at low temperatures where collective effects give rise to a marked deviation form the Debye spectrum.

There are clearly two ways of looking at the origin of the Davidson-Cole expression. First, it arises from cooperativity in the collective orientational relaxation due to the inter-molecular interactions. The second approach is to consider the DC expression to arise from a distribution of relaxation times whose probability distribution is, fortunately, known exactly and is given by

$$
\begin{aligned}
g_{D C}\left(\tau_{D C}\right) & =\frac{\sin (\pi \beta)}{\pi \tau}\left(\frac{\tau}{\tau_{D C}-\tau}\right)^{\beta}(\tau \leqslant 0), \\
& =0 \quad(\tau \geqslant 0) .
\end{aligned}
$$

Rips and Jortner evaluated the average rate by averaging the rate for a given $\tau_{D C}$ over the above expression distribution function. Two important conclusions emerged from their analysis. First, the average electron transfer rate display a fractional dependence of the rate on $\sigma_{D C}$-the exponent being equal to $\beta$-the same exponent that determines the distribution of the inhomogeneously distributed relaxation times. Second, the dependence of the adiabatic rate on the electronic coupling gets modified to $V^{2(1-\beta)}$. Since $\beta=0 \cdot 6$, the dependence of the rate on $V$ is considerably weaker than that in the non-adiabatic limit.

Rips and Jortner did not investigate the time dependence of the reactant population. Such a calculation was carried out by Nadler and Marcus ${ }^{5}$. The decay is nonexponential, particularly at the short times where the rate of Nadler and Marcus agrees with that of Rips and Jortner and predicts the same fractional power-law dependence on $\tau_{D C}$. In contrast, a different behavior holds for the long time rate which is proportional to $\tau_{D C}$. The long time decay is also non-exponential, but markedly less than that at shorter times.

It is interesting to note that one can formulate a dynamical disorder model ${ }^{6}$ of the above problem ${ }^{7}$ where one need not assume a static inhomogeneous distribution of relaxation times, although the dielectric function can still be given by a highly 
non-Debye form like Davidson-Cole. This model is particularly simple for low barrier reactions. Here the equation of motion is given by the one dimensional version of Sumi-Marcus theory but with a time dependent diffusion coefficient the latter is determined by the solvation dynamics time correlation function, as discussed elegantly by Hynes $^{8}$. Note that here the diffusion constant is time dependent because of the non-Debye dielectric function. In a barrierless photoelectron transfer reaction, the short time dynamics is controlled by the time dependence of $D(t)$. Since the latter is determined by the solvation time correlation function, we have a simple way to determine the short time dynamics. In the following we make the above discussion quantitative.

The organization of the rest of the paper is as follows. In the next section we discuss the theoretical formulation. In section III, we discuss the results. Section IV concludes with a brief discussion.

\section{Theoretical formulation}

The potential energy surfaces of the reactant and the product are assumed to be given by the following quadratic forms

$$
\begin{aligned}
& V_{1}(X)=\frac{X^{2}}{4 \lambda_{X}}, \\
& V_{2}(X)=\frac{\left(X-2 \lambda_{X}\right)^{2}}{4 \lambda_{X}}+\Delta G .
\end{aligned}
$$

Here $X$ denotes the solvent coordinate, $\lambda_{X}$ is the solvent reorganization energy ${ }^{1}$ and $\Delta G$ is the free energy change of the reaction. A simple schematic representation of the diabatic surfaces, $V_{R}(X)$ and $V_{P}(X)$, is shown in the figure 1. For low barrier reactions, we shall follow the Sumi-Marcus prescription to include the solvent effects. The reaction coordinate $X$ is the usual Marcus polarization coordinate.

\section{1a. Low barrier reactions}

The time-evolution of the probability distribution of the system on the locally excited surface $\left(P_{1}(X, t)\right)$ is assumed to be given by the following master equation

$$
\frac{\partial P_{1}(X, t)}{\partial t}=\left(\mathscr{L}_{X}-S(X) P_{1}(X, t)+S(X) P_{2}(X, t)\right.
$$

The first term simulates the diffusion in a potential well $V_{1}(X)$. The second and the third terms take into account the actual transfer and the back transfer along the sink curve. $S(X)$ is the position dependent sink function which describes the path along which the electron transfer takes place between the LE and the CT surfaces. In the Sumi-Marcus picture, this sink function describes a wide reaction window when the reorganization energy of the intra-molecular vibrational mode is comparable to that from the solvent polarization and vice versa. The above equation with $P_{1}$ and $P_{2}$ interchanged describes the motion on the CT state in the potential energy surface $V_{2}(X)$.

When the dielectric relaxation is strongly non-Debye, the solvation time correlation function is often biphasic with a large difference between the initial and final time constants. In such a situation the operator $\mathscr{L}$ is a non-Markovian time dependent 


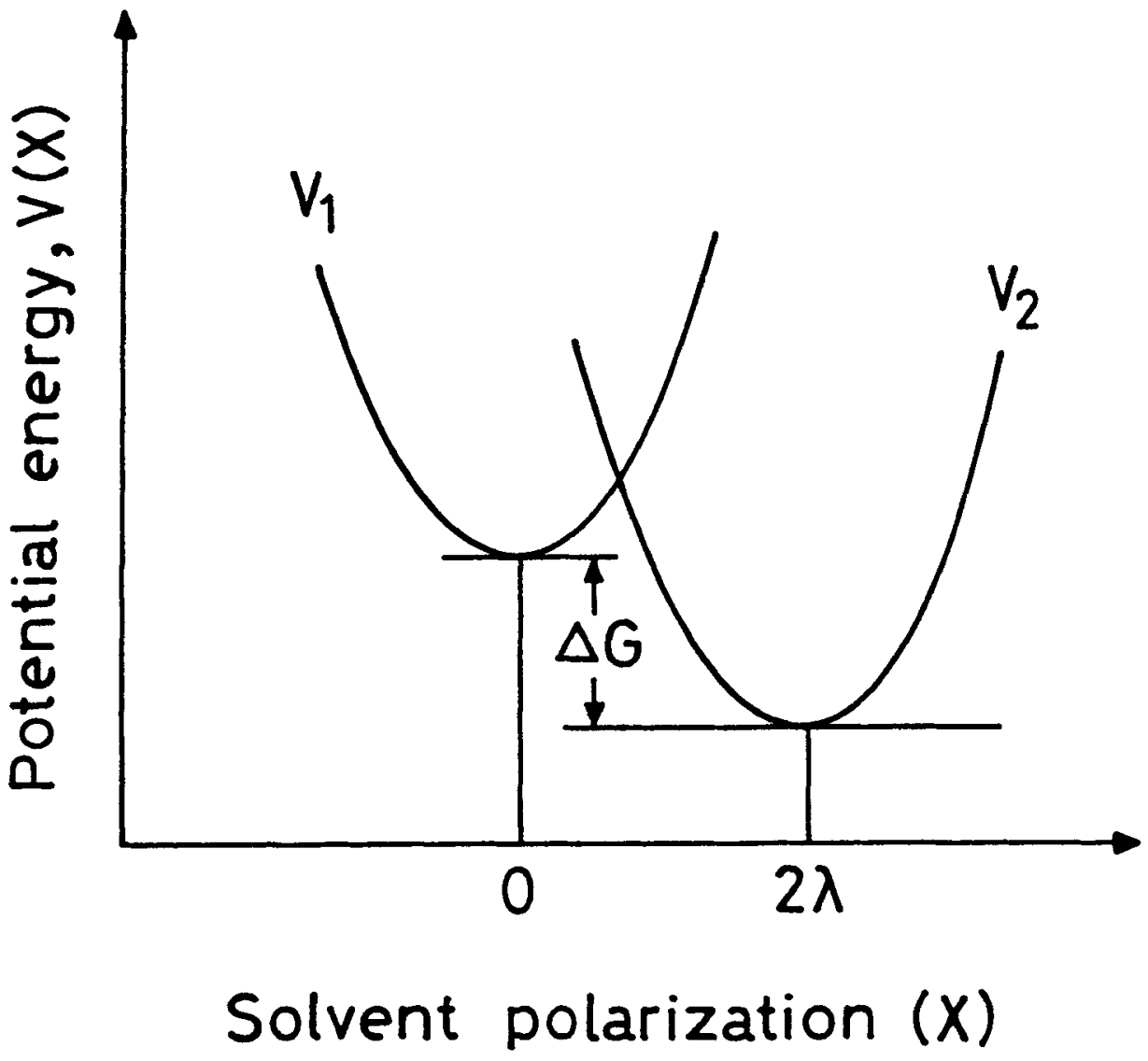

Figure 1. A schematic two surface representation of the free energy surfaces of an electron transfer reaction. The abcissae is the conventional Marcus solvent reaction coordinate, denoted by $\boldsymbol{X}$ in the text. The reorganization energy $(\lambda)$ and the free energy of reaction $(\Delta G)$ are indicated in the figure.

operator. It is assumed to be given by a generalized Smoluchowski operator which is given by the following form

$$
\mathscr{L}_{X}=D_{X}(t)\left(\frac{\partial^{2}}{\partial X^{2}}+\frac{1}{\mathrm{k}_{B} \mathrm{~T}} \frac{\partial}{\partial X}\left[\frac{d V(X)}{d X}\right]\right),
$$

where $D(t)$ is the time dependent diffusion coefficient of motion along the reaction coordinate. $D(t)$ is given by the relation ${ }^{7}$

$$
D(t)=-k_{B} T d \ln \Delta(t) / d t
$$

where $\Delta(t)$ is the solvation time correlation function. Since the liquid is non Debye, $\Delta(t)$ is also non-exponential and, as shown below, may be fitted to a KohlrauschWilliam-Watt (KWW) stretched exponential form ${ }^{8}$

$$
\Delta(t)=\exp \left(-t / \tau_{s}\right)^{\alpha}
$$


where the solvation time $\tau_{s}$ and $\alpha$ are determined by many factors, including the Davidson-Cole exponent, $\beta$. These two exponents $(\alpha$ and $\beta)$ need not be equal, although they may sometimes be close to each other in value.

Equations (5-7) describe the dynamic disorder model. In contrast to the static disorder model, here the solvent relaxation is not quenched.

The experimental observable is the reactant survival probability, $P(t)$, defined by

$$
P(t)=\int_{-\infty}^{\infty} d X P(X, t)
$$

\section{2b. High barrier reactions}

The situation is quite different for the high barrier adiabatic reactions. Here a formulation following Hynes can be used to obtain the long time rate of electron transfer. This scheme, interestingly however, provides a rate not too different from that of Rips and Jortner. For non-exponential $\Delta(t)$ the rate shows fractional dependence on $\tau_{D C}$. In the present model, the solvent dependent rate of electron transfer of an adiabatic reaction is obtained by solving the well-known Grote-Hynes formulae

$$
\begin{aligned}
& k_{e t}=\left(\lambda_{r} / \omega_{b}\right) k^{T S T}, \\
& \lambda_{r}=\frac{\omega_{b}^{2}}{\lambda_{r}+\zeta\left(\lambda_{r}\right)}, \\
& \Delta(z)=\frac{1}{z^{2}+\omega_{R}^{2}+z \zeta(z)},
\end{aligned}
$$

where $k^{T S T}$ is the transition state rate of the bi-stable (double well) system with a barrier frequency $\omega_{b}$, reactant well frequency $\omega_{R}$ and an activation energy which is determined by the Marcus theory. In the above equation, $\lambda$, is the reactive frequency, $\zeta(z)$ is the frequency dependent reactive friction and $\Delta(z)$ is the Laplace transform of the solvation time correlation function $\Delta(z)=\int_{0}^{\infty} d t \exp (-z t) \Delta(t)$. The solvent dependence is determined by the curvature of the adiabatic surface near the barrier top. This curvature is determined by $\omega_{b}$ which is turn is determined by the electronic coupling $V_{e l}$ between the two surfaces. For harmonic diabatic surfaces, this relation between the barrier curvature $\left(\omega_{b}\right)$ and the electronic coupling is given by the following simple relation

$$
\omega_{b}^{2}=\omega_{R}^{2} \cdot\left[1+\lambda / V_{e l}\right]
$$

The above equation shows that we have a cusp-like barrier for a weakly adiabatic reaction and a broad barrier for an adiabatic reaction. It is curious to note that in this model, the dynamic solvent effects also depend on the solvent reorganization energy $(\lambda)$ through the ratio $\lambda / V_{e l}$. We shall show below that this has an important role to play in determining the dynamic solvent effect and is in fact connected to a previous observation of Rips and Jortner.

We next present the results.

\section{Results and discussion}

In order to obtain the time dependent reactant survival probability, we first need to calculate the solvation time correlation function, $\Delta(t)$. We have calculated this $\Delta(t)$ for 


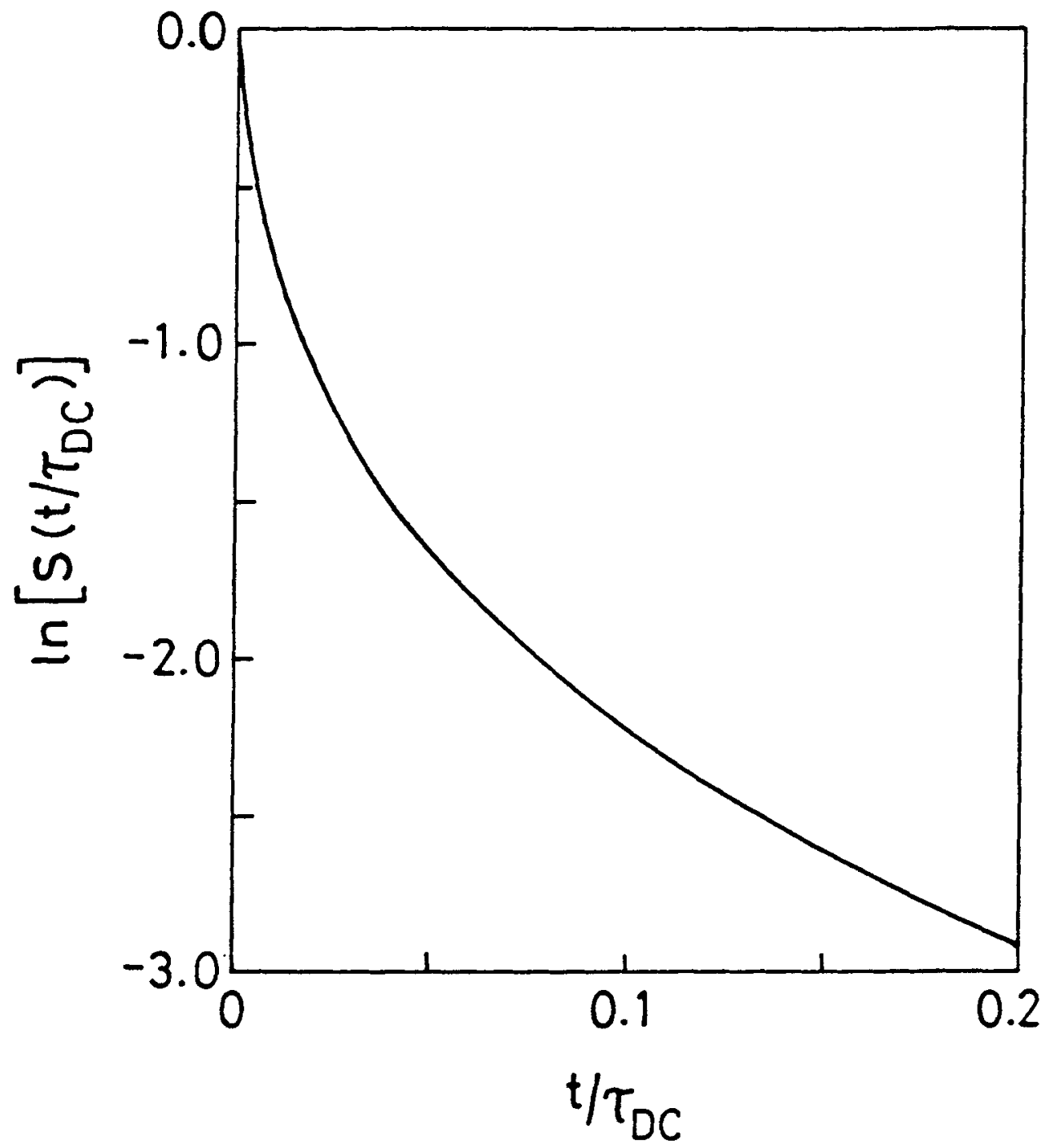

Figure 2. The calculated normalized solvation time correlation function, $\Delta(t)$ is plotted against time $(t)$ for solvation of a spherical solute probe in solvent glycerol at temperature $T=233 \mathrm{~K}$. The solute probe is chosen to be of the same size as the well-known dye molecule Coumarin 103. This solvation time correlation function has been calculated by using the theory described in references 10 and 11 .

glycerol at low temperature $(T=233 \mathrm{~K})$ where the dielectric relaxation can be fitted to the Davidson-Cole form with $\varepsilon=60 \cdot 5, \varepsilon_{\infty}=4 \cdot 2, \tau_{D C}=1 \cdot 15 \cdot 10^{-5} \mathrm{~s}$ and $\beta=0.608$. The normalized solvation time correlation function $(\Delta(t))$ has been obtained by using the molecular theory which has been discussed elsewhere in detail ${ }^{10}$ and which has been remarkably successful in describing solvation dynamics in both simple and complex systems. The results of the calculation of $\Delta(t)$ are shown in figure 2 . The decay is clearly non-exponential. 


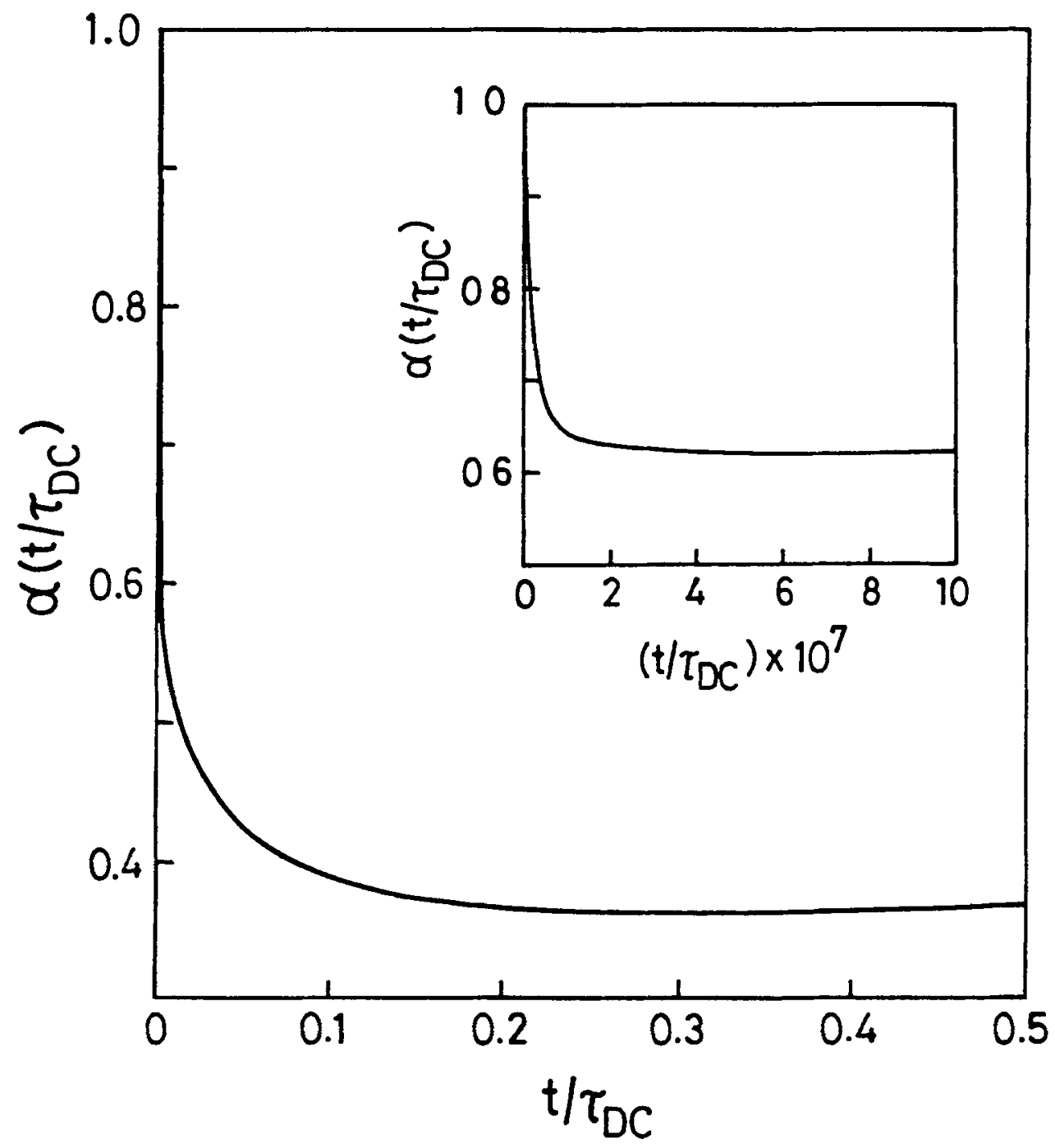

Figure 3. The time dependence of the stretched exponential exponent (the KWW exponent) $\alpha(t)$ is plotted against the scaled time $t / \tau_{D C} \cdot \alpha(t)$ has been calculated using (14) of the text. The inset depicts the same for a very short time.

In order to make connection with the work of Rips and Jortner, we need to investigate the nature of the non-exponentiality by calculating the time dependence of the stretched-exponential exponent $\alpha$, defined by

$$
\alpha(t)=-\frac{d}{d t} \ln (-\ln \Delta(t))
$$

The result of this calculation is shown in figure 3. In the same figure very short time behavior is depicted in the inset. There are two points to note. First, in the long time we find a good stretched-exponential behavior of $\Delta(t)$ with the $\mathrm{KWW}$ exponent ${ }^{9} \alpha$ having 
a value close to $0 \cdot 38$. In the short time, however, we find a different behavior. Here we find a stretched exponential behavior with the value of the KWW exponent equal to 0.6. The above results have the following consequence in electron transfer.

When the electron transfer reaction is strongly coupled to solvation dynamics, then in the short time one may expect a non-exponential reaction dynamics which is controlled largely by the time dependent diffusion coefficient, $D(t)$, related to the solvation time correlation function $\Delta(t)$ by $(7)$. When $\Delta(t)$ is stretched exponential, $D(t)$ has a fractional dependence on the relaxation time constant which is here proportional to the Davidson-Cole relaxation time, $\tau_{D C}$. This part agrees with the prediction of Rips and Jortner. However, the present results seem to differ in detail from the prediction of Nadler and Marcus in the sense that $\mathrm{N}-\mathrm{M}$ predicts an exponential rate in the longtime that seems unlikely unless the intersystem crossing rate is much slower than the solvation dynamics which is doubtful in slow liquids like glycerol.

We next turn to calculate the rate and for this we need to specify the coupling between the two surfaces and the nature of the sink, $S(X)$. One can derive a close form, useful expression in the limit when the coupling is strong (the adiabatic limit) and the sink is narrow. Here for an arbitrary initial distribution, $P_{0}(X)$, the reactant population is given by the following expression

$$
P(t)=\operatorname{erf}(F(X, t)) \text {. }
$$

The function $F(X, t)$ is given by the following expression

$$
F(X, t)=\frac{1}{\sqrt{2 \pi\left(1-\Delta^{2}(t)\right)}} \exp \left[-\left(X-X_{0} \Delta(t)\right)^{2}\right] /\left[2\left(1-\Delta^{2}(t)\right)\right] .
$$

Several interesting predictions follow straightaway. First, in the long time, the electron transfer kinetics is determined by the solvation time correlation function, $\Delta(t)$. Second, in the short time, the reaction is even slower than $\Delta(t)$

When the initial distribution is an equilibrium distribution on the reactant surface, then the following simple expression is obtained

$$
P(t)=(2 / \pi) \operatorname{Sin}^{-1}(\Delta(t)) \text {. }
$$

Note that this simple expression holds even when the solvent relaxation is nonexponential-earlier derivations, for example by Sumi and Marcus ${ }^{1}$, were restricted to exponential $\Delta(t)$ only.

The time dependence of the reactant population given by the above equation is shown in the figure 4 calculated with the $\Delta(t)$ for glycerol which has already been depicted in figure 2. In order to compare and understand the correlation with solvation dynamics we also show the solvation time correlation function itself. Note the slaving of the electron transfer reaction to the solvation dynamics in the long time. But the two can be rather different at the very short time which, however, may not be noticeable. These results are in qualitative agreement with the earlier studies ${ }^{4,5}$, except that (17) predicts strongly non-exponential dynamics even in the long time. This is a direct consequence of assuming an absorbing barrier at the minimum ("the pinhole sink"). In general, sink function is much more complex. It can be wide because of the participation of the non-reactive vibrational modes ${ }^{1}$ and will have a finite rate. Thus, the decay of the reactant probability would be less slaved to solvation dynamics than predicted in the figure 4. Unfortunately, for the general case, no simple solution is available and the solution needs to be obtained numerically. 


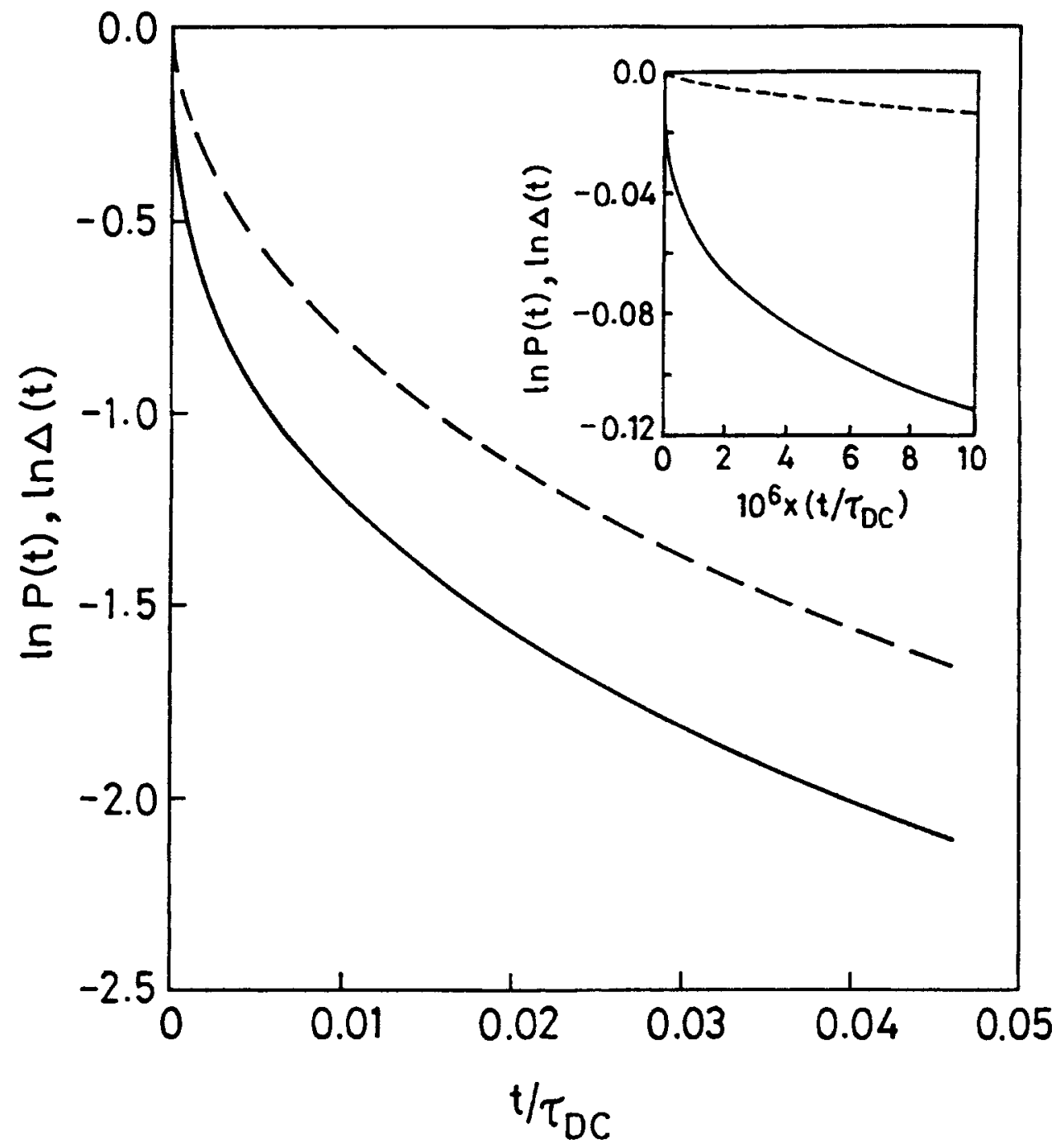

Figure 4. The reactant survival probability function $P(t)$ given by $(17)$ is plotted against time for an adiabatic electron transfer reaction (the solid line). For comparison, we have also plotted the normalized solvation time correlation function, $\Delta(t)$ in the same figure (the dashed line). In the inset, we show the two functions in the limit of a very short time.

For high barrier reactions, the barrier frequency could be rather large in the weakly adiabatic limit. In this limit, solvent dynamics will have little effect on the electron transfer rate which will be given by the transition state theory result, that is, by the Marcus expression. In the adiabatic limit, on the other hand, the rate can be substantially lower than the TST prediction and the extent of the decrease will certainly be determined by the coupling element $V_{e l}$, for a given value of the Marcus reorganization energy, $\lambda$. An interesting consequence of (13) is that the barrier frequency $\omega_{b}$ of an adiabatic reaction varies as $\left[1+\lambda / V_{e l}\right]^{0.5}$. This observation may be useful for a weakly 
adiabatic reaction where $\omega_{b}$ is predicted to vary as $V_{e l}^{-0.5}$. Because solvent dielectric frictional retardation increases as $\omega_{b}$ decreases, rate of a weakly adiabatic electron transfer reaction will show complex dependence on the electronic coupling parameter, $V_{e l}$. In general, the dependence will be weaker than $V_{e l}^{2}$, as already observed by Rips and Jortner. In addition, the dynamic solvent effect will introduce a coupling between the nonexponentiality parameter $(\alpha)$ of the solvation time correlation function, $\Delta(t)$ and the electron transfer rate. Another intersting point is that the extent of this coupling depends on the reorganization energy $\lambda$. While this is expected on physical grounds, we are not aware of any theoretical study addressing this point which deserves to be studied further.

\section{Conclusion}

Broadly, there are two different causes of marked non-exponentiality in electron transfer kinetics. The existence of the reaction system in multiple static configurations is the common explanation and this is certainly valid in many cases, especially in biological systems. This is also the Rips-Jortner-Nadler-Marcus scenario where the reaction occurs in a system characterized by a distribution of dielectric relaxation times which are uncorrelated among themselves (quenched disorder model). The second explanation is presented here where we have a dynamic disorder but with a high degree of non-exponentiality. We have shown here that in this case one can calculate the solvation time correlation function from the non-Debye dielectric relaxation data. The resulting non-exponential solvation time correlation function defines a time dependent diffusion constant for the motion on the solvent reaction energy surface. It is shown that many of the results of Rips-Jortner-Nadler-Marcus analysis can be recovered in this dynamic disorder model. These are, however, some notable differences. The most important is that the present treatment predicts larger non-exponentiality in the long time. In a future publication we shall address the details of the difference between the static and the dynamic disorder models ${ }^{11}$.

\section{Acknowledgements}

I thank Mr Ranjit Biswas for help and discussions during the course of this work. The work was supported in part by the Department of Science and Technology, Council of Scientific and Industrial Research and the Indo-French Center for Promotion of Advanced Research, New Delhi.

\section{References}

1. Sumi H and Marcus R A 1986 J. Chem. Phys. 844272

2. McGuire $M$ and McLendon G 1986 J. Phys. Chem. 90, 2549

3. Davidson D W and Cole R H, 1951 J. Chem. Phys. 19, 1484; ibid, 18, 14171950

4. Rips I and Jortner J, 1987 Chem. Phys. Lett. 133411

5. Nadler W and Marcus R A 1988 Chem. Phys. Lett. 14424

6. Zwanzig R 1990 Acct. Chem. Res. 23148

7. Bagchi $\mathrm{B}$, unpublished work

8. Hynes J T 1986 J. Phys. Chem. 90, 3701

9. Kohlrausch R 1847 Pogg. Ann. 12 393; Williams G and Watts D C 1966 Trans. Faraday Soc. 6680

10. Biswas R and Bagchi B 1996 J. Phys. Chem. 1004278

11. Biswas $R$ and Bagchi $B$ to be published 